\title{
Effect of Nucleases on the Cellular Internalization of Fluorescent Labeled DNA-Functionalized Single-Walled Carbon Nanotubes
}

\author{
Hye Kyung Moon ${ }^{1}$, Chan II Chang ${ }^{2}$, Dong-Ki Lee ${ }^{2}(\bowtie)$, and Hee Cheul Choi ${ }^{1}(\bowtie)$ \\ ${ }^{1}$ Department of Chemistry, Pohang University of Science and Technology (POSTECH), San 31, Hyoja-Dong, Nam-gu, Pohang 790-784, \\ Korea \\ ${ }^{2}$ Department of Chemistry, Sungkyunkwan University, Natural Science Campus, Suwon 440-746, Korea \\ Received: 8 July 2008/ Revised: 16 August 2008/ Accepted: 3 September 2008 \\ CTsinghua Press and Springer-Verlag Press. This article is published with open access at Springerlink.com
}

\begin{abstract}
Nuclease effects on the cell internalization of single-walled carbon nanotubes (SWNTs) functionalized with fluorescent-labeled DNA in serum containing cell growth media were examined. When Cy3-labeled DNAfunctionalized SWNT conjugates (Cy3DNA-SWNTs) were incubated with HeLa cells in a fatal bovine serum (FBS) medium, a high fluorescence intensity was obtained from the cells, indicative for the high level inclusion of Cy3DNA-SWNTs. However, the fluorescence intensity was remarkably reduced if Cy3DNASWNTs were incubated with cells in the FBS-free medium. Further systematic control experiments revealed that $\mathrm{Cy} 3$ dye molecules were released from Cy3DNA-SWNT conjugates by nuclease, and the free Cy3 dyes penetrate into HeLa cell with high efficiency. Although the actual amounts of SWNTs internalized in the cells were almost identical for both cells incubated in the FBS-present and FBS-absent media according to the Raman measurements, one should be cautious to determine the degree of SWNT internalization based on the fluorescence intensities especially when the coloring dye molecules were linked to oligonucleotides in nuclease containing media.
\end{abstract}

\section{KEYWORDS}

Single-walled carbon nanotube, oligonucleotide, cellular delivery, molecular transporter, nuclease

\section{Introduction}

The development of smart biocompatible nanomaterials for target-specific disease therapeutics is of great interest for early disease detection as well as efficient cell apoptosis detection in cell-based biomedical studies [1-3]. Recently, zero- (0D) and onedimensional (1D) nanomaterials have been widely investigated for these purposes since their quantum size effect-based unique electrical and colorimetric properties are suitable for the fabrication of nanoelectronic transistors, sensors, and platforms for various biomolecules and drug delivery systems [46]. Among many promising candidates, single-walled carbon nanotubes (SWNTs) have been considered not only as an efficient molecular transporter but also as a thermotherapeutic agent on the basis of their optothermic effects [7-10]. For example, SWNTs have

Address correspondence to Hee Cheul Choi, choihc@postech.edu; Dong-Ki Lee, dklee@skku.edu 
been shown to act as an efficient cellular transporter in living systems [11-13]. In particular, nucleic acid molecules such as DNA, RNA, and siRNA have been conjugated with SWNTs by various strategies [14-17] and successfully delivered into living cells $[18,19]$.

Cell permeability is one of many essential prerequisites if nanomaterials are to become an efficient molecular transporter. In general, fluorescent dye molecules are commonly conjugated onto the surface of nanomaterials along with other guest molecules (DNA, siRNA, etc.) for systematic studies of their cell permeability, and the extent of penetration of the conjugated nanomaterials into target cells is generally determined by counting the fluorescence intensity i.e., the amount of internalized conjugated nanomaterial is assumed to be directly proportional to the fluorescence intensity. However, care is required especially when such cell permeability tests are conducted under serumcontaining incubation conditions because the many active nucleases existing in most cellular growth media (serums) readily digest oligonucleotides very efficiently [20]. This may cause the separation of dye molecules from nanomaterials, hence leading to misleading values of the efficiency of internalization of the nanomaterials.

Herein we report that the amount of SWNTs functionalized with fluorescent $\mathrm{Cy} 3$ dye-labeled DNA (Cy3DNA-SWNTs) internalized into HeLa cells shows a significant variation with incubation environment. While high fluorescence intensities are always observed when cells are incubated with Cy3DNA-SWNTs in media containing nucleases that are commonly present in a conventional cell growth medium, the fluorescence intensities are substantially decreased when such nucleases are absent.

\section{Experimental}

\subsection{Preparation of Cy3DNA-SWNT complex and characterization}

HiPCO SWNTs purchased from CNI (Carbon Nanotechnologies, Inc., USA) were mixed with $10 \mu \mathrm{mol} / \mathrm{L}$ Cy3 labeled single-stranded DNA in aqueous solution to form a Cy3DNA-SWNT suspension $(250 \mathrm{mg} / \mathrm{L})$. The DNA sequence was
Cy3-TGGACAAGTGGAATG (Bioneer Corporation, Korea) [10]. The mixture of Cy3DNA and SWNTs was then sonicated for $45 \mathrm{~min}$ to $1 \mathrm{~h}$ in a bath sonicator (Fisher Scientific FS60 ultrasonic cleaner, operating at $42 \mathrm{kHz}$ ), then centrifuged at $13000 \mathrm{rpm}$ for $3-4 \mathrm{~h}$ to remove aggregates and bundles. This process was repeated three times before the final supernatant (1 $\mathrm{mL}$ ) was acquired. The final supernatant contains Cy3DNA-SWNTs with small amounts of free Cy3DNA. In order to remove free Cy3DNA, the mixture was filtered through a polycarbonate filter (Whatman, pore size $=100 \mathrm{~nm}$ ) by vacuum filtration, and then re-suspended in $1 \mathrm{~mL}$ sterilized water by sonication. This filtration/re-suspension process was repeated three times. The filtrates from each step, as well as the final Cy3DNA-SWNT suspension were examined by UV-vis spectroscopy (absorbance measured at 260 and $550 \mathrm{~nm}$, Agilent 8453 spectrophotometer) to confirm the removal of free Cy3DNA. The final Cy3DNA-SWNT suspension was further characterized by atomic force microscopy (AFM, tapping mode, Nanoscope IIIa, Digital Instruments) and UV-vis-NIR spectrometry (UV-3600 spectrometer, Shimadzu) to confirm the successful conjugation of Cy3DNA onto SWNTs. For AFM analyses, $60 \mu \mathrm{L}$ of SWNT suspension was dispersed and spun on $\mathrm{SiO}_{2}$ substrate, then thoroughly rinsed with pure water and dried with a $\mathrm{N}_{2}$ stream.

\subsection{Cell culture and cellular incubation}

The HeLa cells were cultured in Dulbecco's modified Eagle's medium (DMEM) supplemented with $10 \%$ fetal bovine serum (FBS) and $1 \%$ penicillinstreptomycin (all reagents from Hyclone) at $37^{\circ} \mathrm{C}$ in a humidified atmosphere with $5 \% \mathrm{CO}_{2}$. The HeLa cells were seeded on a glass cover slip in 12-well plates and incubated for $18 \mathrm{~h}$ at a density of $2 \times 10^{5}$ cells per well in $1 \mathrm{~mL}$ of medium. The medium in each well was replaced with fresh media having $10 \%$ FBS or without FBS depending on the experiment being undertaken before cellular incubation. For systematic studies of the nuclease effect on the internalization of SWNTs, solutions of 1) Cy3DNA (1 $\mu \mathrm{mol} / \mathrm{L}), 2)$ Cy3 dye (1 $\mu \mathrm{mol} / \mathrm{L}$ ), and 3) Cy3DNA-SWNT (ca. $25 \mathrm{mg} / \mathrm{L}$ ) were added to wells containing 1) FBS-containing medium, 2) FBS-free medium, and 3) nuclease (S1 Nuclease, 
Sigma-Aldrich, 100 times diluted) with FBS-free medium, and then incubated for $12 \mathrm{~h}$ at $37{ }^{\circ} \mathrm{C}$ in a $5 \%$ $\mathrm{CO}_{2}$ atmosphere. After incubation, the cell medium was removed from each well which was then rinsed thoroughly with phosphate buffer saline (PBS).

\subsection{Fluorescence and confocal microscopy}

The fluorescence from each cell mounted on a glass slide was characterized using a fluorescence microscope (Nikon Eclipse 80i). Three cellular images were selected for each case, and the average fluorescence intensity of cells was determined by using cellular image analysis software (i-solution, InnerView Co., Korea). For statistical analyses, a Student's t-test was used and a 95\% confidence limit was taken to be significant (defined as $P<0.05$ ). The results were expressed as arithmetic means \pm s.e. (standard error). Cross-section imaging was performed by confocal microscopy (Bio-Rad Radiance 2100 confocal microscope, Bio-Rad, Inc., UK) with laser excitation of $543 \mathrm{~nm}$ and emission filters (560-610 nm). Sectioned images were collected at $2.5 \mu \mathrm{m}$ intervals.

\subsection{Flow cytometry measurements}

Analyses of the fluorescence intensity from individual cells were carried out by flow cytometry. The HeLa cells in a 6-well plate with $5 \times 10^{5}$ cells / well were pre-incubated in $10 \%$ FBS and $1 \%$ penicillin-streptomycin for $18 \mathrm{~h}$ at $37{ }^{\circ} \mathrm{C}$ in a $5 \% \mathrm{CO}_{2}$ atmosphere. Upon completion of the designated series of experiments, the cells were washed thoroughly with PBS, then trypsinized and resuspended in PBS for analysis using a fluorescence activated cell sorter (FACSCalibur, Becton Dickinson). Cells were analyzed by detecting emission at $585 \mathrm{~nm}$ with a 585/42 bandpass filter (excitation wavelength of $488 \mathrm{~nm}$ ). For each case, 10000 cells were counted and analyzed with CellQuest software. Fluorescence histograms were obtained by following the typical FACS protocol.

\subsection{Raman measurements of Cy3DNA-SWNTs in cells}

Since SWNTs display a strong characteristic resonance Raman band at around $1590 \mathrm{~cm}^{-1}$ (G band) [21], the amounts of free SWNTs in solution and
SWNTs internalized in cells were directly determined by measuring $\mathrm{G}$ band intensities. Pre-incubated HeLa cells on a cover slip were treated with Cy3DNASWNTs (ca. $25 \mathrm{mg} / \mathrm{L}$ ) in FBS-free and 10\% FBS media. After incubation for $12 \mathrm{~h}$, cells were thoroughly washed with PBS and then cell-seeded cover slips were placed on a glass slide for measurements. The G band intensities from SWNTs internalized in cells were measured at $1590 \mathrm{~cm}^{-1}$ using a Raman spectrometer (laser excitation wavelength of $785 \mathrm{~nm}$, $100 \mathrm{~mW}$ power, 50×objective, $10 \mathrm{~s}$ collection time, 1 scan, SENTERRA Dispersive Raman Microscope, Bruker). Nine randomly selected cells in each sample were measured for statistical analyses.

\section{Results and discussion}

\subsection{Synthesis of Cy3DNA-SWNT conjugates}

A highly stable aqueous suspension of Cy3DNASWNT was prepared via noncovalent adsorption of Cy3DNA on SWNTs. Although SWNTs and Cy3DNA are not miscible at all when they are mixed, the SWNTs were mechanically dispersed in the aqueous solution of Cy3DNA by ultrasonic treatment for up to $1 \mathrm{~h}$. During the preparation of Cy3DNASWNT conjugates, attention was paid to ensure two important prerequisites for accurate systematic studies of the internalization of Cy3DNA-SWNTs into cells were met: 1) free Cy3DNA should be absent in the final Cy3DNA-SWNT suspension; 2) Cy3DNASWNTs should exist in individual form without being bundled. Firstly, the removal of the free Cy3DNA was accomplished by repeated filtrations of the reaction mixture. As shown in Fig. 1(a), two strong absorption peaks at 260 and $550 \mathrm{~nm}$ corresponding to aromatic bases of DNA and Cy3 dye from Cy3DNA, respectively, were observed in the filtrate from the first filtration. However, both peak intensities were drastically reduced in the filtrate from the second filtration, indicating that free Cy3DNA molecules had been essentially completely removed from the solution. Moreover, the final Cy3DNA-SWNT suspension is sufficiently stable to exist for longer than two months at room temperature without any severe aggregation or precipitation (Fig. 1(b))

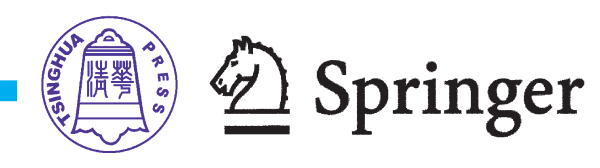


[10]. Secondly, the AFM image of Cy3DNA-SWNTs dispersed on a $\mathrm{SiO}_{2}$ substrate indicates that Cy3DNASWNTs are almost completely debundled (Fig. 1(d)). The average diameter of the Cy3DNA-SWNTs is ca. $2.8 \mathrm{~nm}$ [22], which confirms the successful coating of Cy3DNA on bare SWNTs with average diameter of ca. $1.5 \mathrm{~nm}$ [23]. Meanwhile, the intrinsic electronic properties of SWNT are not significantly changed after conjugation with Cy3DNA as most of the distinctive electronic transitions were retained, as indicated by UV-vis-NIR spectroscopy (Fig. 1(c)) [24, 25].

\subsection{The effect of incubation medium environment} on the internalization of Cy3DNA-SWNTs into cells

In order to examine the effect of the incubation medium on Cy3DNA-SWNTs prior to the cellular incubation tests, Cy3DNA-SWNTs were first exposed to DMEM medium containing 10\% fetal bovine serum (FBS) and the same medium without FBS. As the incubation time increased, some aggregates and precipitate of SWNTs were formed in the $10 \%$ FBS-containing medium. As determined from the absorbance intensity at $550 \mathrm{~nm}$, much larger amounts of Cy3DNA were released from SWNT backbones when Cy3DNA-SWNTs were exposed to the $10 \%$ FBS-containing medium than was the case when Cy3DNA-SWNTs were exposed to the FBS-free medium (Fig. S-1 in the Electronic Supplementary Material (ESM)). Note that the overall spectra of Cy3DNA SWNTs in FB-free medium show higher intensity due to mixed SWTNs and Cy3DNA.

Next, Cy3DNA-SWNTs were treated with HeLa cells in three different incubation media in order to investigate the effect of incubation medium on the internalization of SWNTs into the cells: (1) $10 \%$ FBS-supplemented DMEM medium, (2) FBS-free DMEM medium, and (3) S1 nuclease added to FBSfree DMEM medium. After incubating HeLa cells with Cy3DNA-SWNTs in a 10\% FBS-supplemented DMEM medium that is conventionally used for
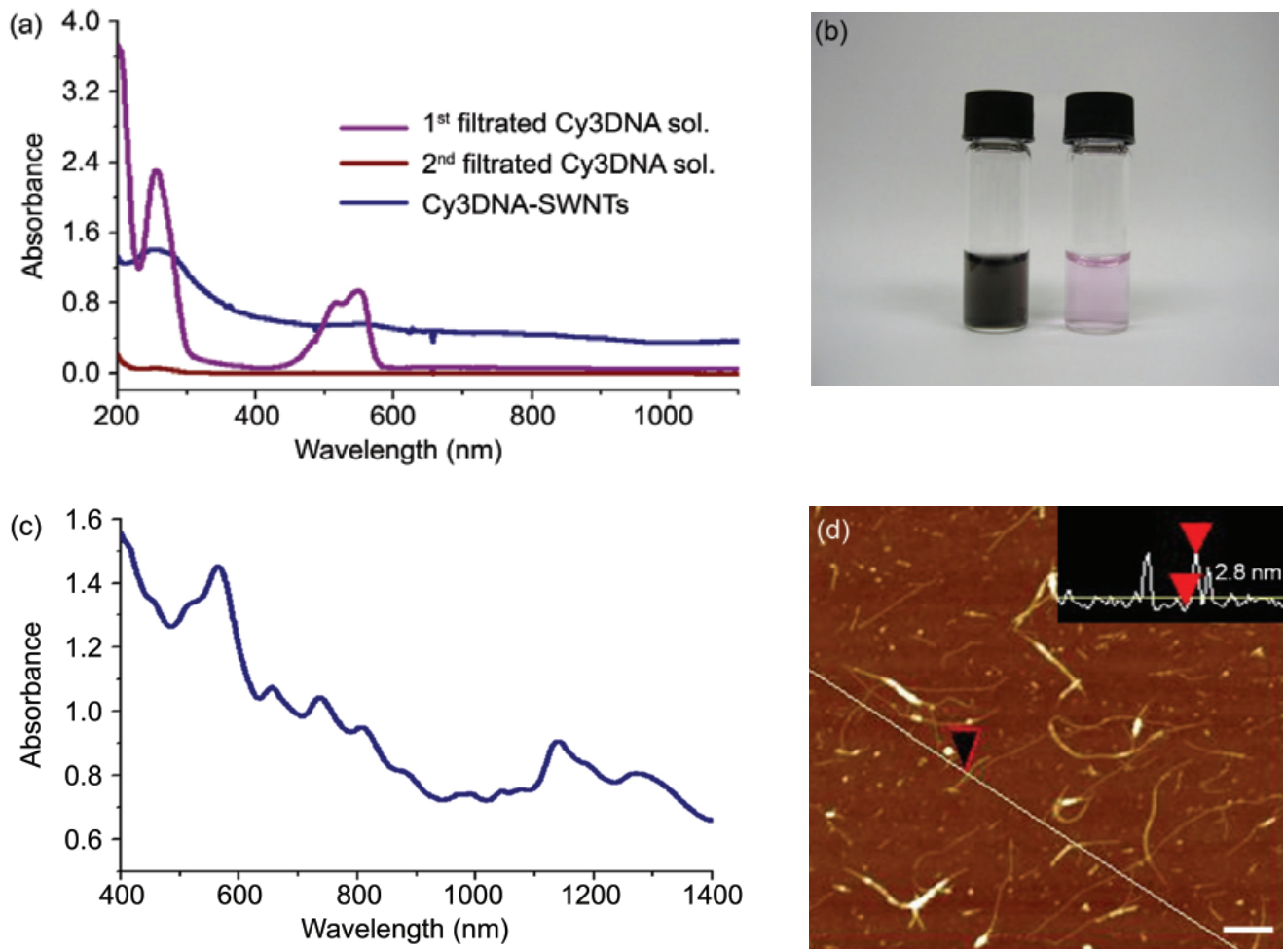

Figure 1 (a) UV-vis spectra of filtrates from the preparation of Cy3DNA functionalized SWNT containing free Cy3DNA (violet $-1^{\text {st }}$ filtration, red $-2^{\text {nd }}$ filtration) and final Cy3DNA functionalized SWNT suspension after repeated filtration (blue). (b) Photograph of a Cy3DNA-SWNT suspension (left) and the filtrate from the $1^{\text {st }}$ filtration containing free Cy3DNA (right). (c) UV-vis-NIR spectrum of an individual Cy3DNA-SWNT (the peak at $\sim 550 \mathrm{~nm}$ corresponds to Cy3 dye molecules tagged on DNA). (d) Atomic force microscopy (AFM) image of Cy3DNA-SWNTs dispersed on $\mathrm{SiO}_{2}$. The scale bar is $200 \mathrm{~nm}$ 
normal cell growth, high fluorescence intensity was detected from HeLa cells (Fig. 2(a)). At first sight, this suggests that significant amounts of Cy3DNASWNTs have been successfully internalized in HeLa cells. In contrast, when we examined internalization of Cy3DNA-SWNTs into HeLa cells in an FBSfree DMEM medium, the fluorescence intensity was significantly decreased, by ca. $70 \%$ (Fig. 2(b)). In order to further confirm the effect of nucleases on the fluorescence intensity, Cy3DNA-SWNTs were incubated in an FBS-free medium to which S1 nucleases had been added. In this case, the fluorescence intensity (Figs. 2(c) and 2(d)) is almost comparable to the one observed in the presence of FBS shown in Fig. 2(a).

According simply to the fluorescence intensity differences, it seems that nucleases control the amounts of Cy3DNA-SWNTs internalized into HeLa cells because the fluorescence intensity is much lower when the cells are incubated in the absence of nucleases (i.e., in an FBS-free medium) than when incubated in the presence of nucleases. However the origin of the fluorescence should be more carefully considered, especially when the incubation medium contains nucleases, because nucleases readily fractionate DNA. In other words, any nanomaterial involving SWNTs conjugated with DNA or DNA-linked functional molecules has a high chance of losing the conjugation in the presence of nucleases, resulting in separation of the dye or functional molecules from DNA and eventually from the nanomaterial. Since the 10\% FBSsupplemented DMEM medium contains significant amounts of nucleases [20], the Cy3Dye-DNA-SWNT conjugation system also might be broken. Therefore, fluorescence does not solely represent internalization of Cy3DNA-SWNTs, but also includes internalization of either released free $\mathrm{Cy} 3$ dyes or Cy3DNA fragments partially digested by nucleases. This led us to examine incubation of Cy3DNA and Cy3 dye molecules into HeLa cells in order to be sure of the origin of
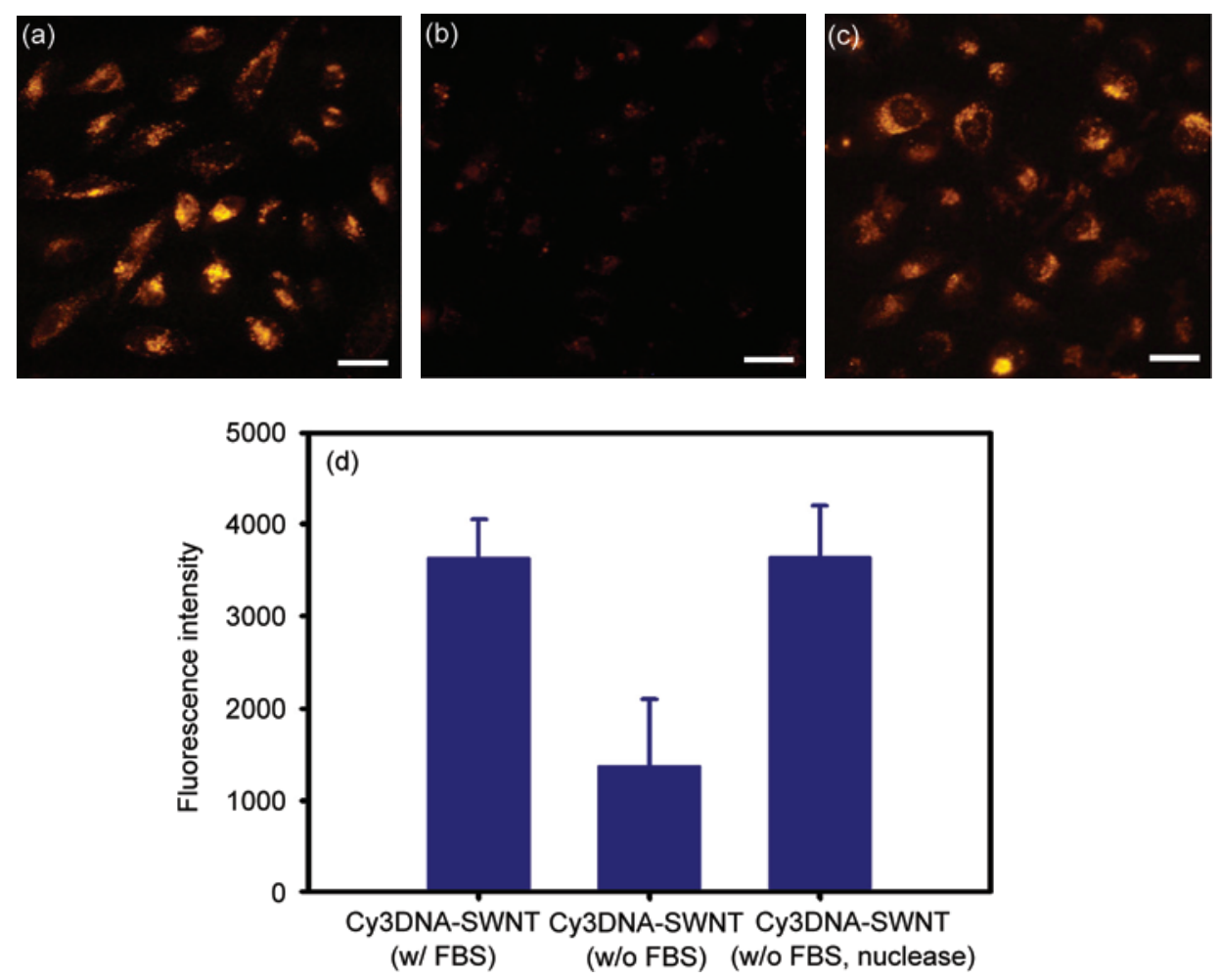

Figure 2 Fluorescence microscopy images of HeLa cells after incubation of Cy3DNA-SWNTs in (a) 10\% FBS-supplemented DMEM, (b) FBS-free DMEM, and (c) FBS-free DMEM with added S1 nuclease. The scale bar is $20 \mu \mathrm{m}$. (d) Fluorescence histogram for incubation of Cy3DNA-SWNTs in $10 \%$ FBSsupplemented DMEM, in FBS-free DMEM, and in FBS-free DMEM with added S1 nuclease (arithmetic means \pm s.e., $n=3$ ). When the medium does not contain nucleases (i.e., no FBS or S1 nuclease), there is a reduction in fluorescence intensity of $\sim 70 \%$ (Student's t-test; $P<0.05$ )

(1)

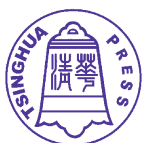

算 Springer 
fluorescence. As shown in Fig. 3, cells incubated with Cy3DNA in the presence of FBS show much higher fluorescence intensity (Fig. 3(a)) compared to those incubated in an FBS-free DMEM medium (Fig. 3(b)). From these results, it can be confirmed that Cy3DNA molecules are internalized only in the presence of FBS, indicating that Cy3DNA is further digested by nucleases to release free $\mathrm{Cy} 3$ dyes that are subsequently internalized into HeLa cells (Scheme 1). Note that the cross-section images of the cells obtained by confocal fluorescence microscopy also confirm that the fluorescence originated from inside the cell rather than from the exterior surfaces of the cell, where free Cy3 dyes might be adsorbed nonspecifically (Figs. 3(d) and 3(e)). Further evidence in support of this mechanism comes from the fact that highly intense fluorescence was detected when HeLa cells were incubated with $\mathrm{Cy} 3$ dye, regardless of the presence or absence of FBS (Figs. 4(a), 4(b) and $4(\mathrm{c})$ ), showing that free $\mathrm{Cy} 3$ dye molecules readily penetrate into $\mathrm{HeLa}$ cells. Note that $\mathrm{Cy} 3$ dye is chemically inert to nucleases, and contains hydrophobic moieties enabling its internalization into HeLa cells. Moreover, the effect of nucleases on the fragmentation of dye molecules from DNA-SWNTs is not specific to Cy3, and is also observed with other commonly used dyes including Cy5 and fluorescein (Fig. S-2 in the ESM).

Furthermore, the possibility of internalization of Cy3DNA-SWNTs into cells via nonspecific adsorption of proteins on the sidewalls of SWNTs was excluded, although it is known that protein adsorption on nanoparticles readily facilitates their internalization into cells $[26,27]$. When the cellular uptake of Cy3DNA-SWNTs in the presence of bovine serum albumin (BSA) $(0.5-2.0 \mathrm{mg} / \mathrm{mL})$ was examined as a function of time, BSA did not expedite the cellular uptake even when the concentration was $2.0 \mathrm{mg} / \mathrm{mL}$ (Fig. S-3 in the ESM). On the other hand, HeLa cells incubated with Cy3DNA-SWNTs in an FBS-free medium with added S1 nuclease showed gradual increases in fluorescence intensities analogous to those observed with the FBS-containing medium. It was also observed that on addition of BSA, a homogeneous suspension of Cy3DNA-SWNT was maintained whereas agglomerates were formed on addition of S1 nuclease to a Cy3DNA-SWNT suspension (Fig. S-3 insets, in the ESM)).

Although fluorescence microscopy provides both fluorescence intensity and cellular shape information,
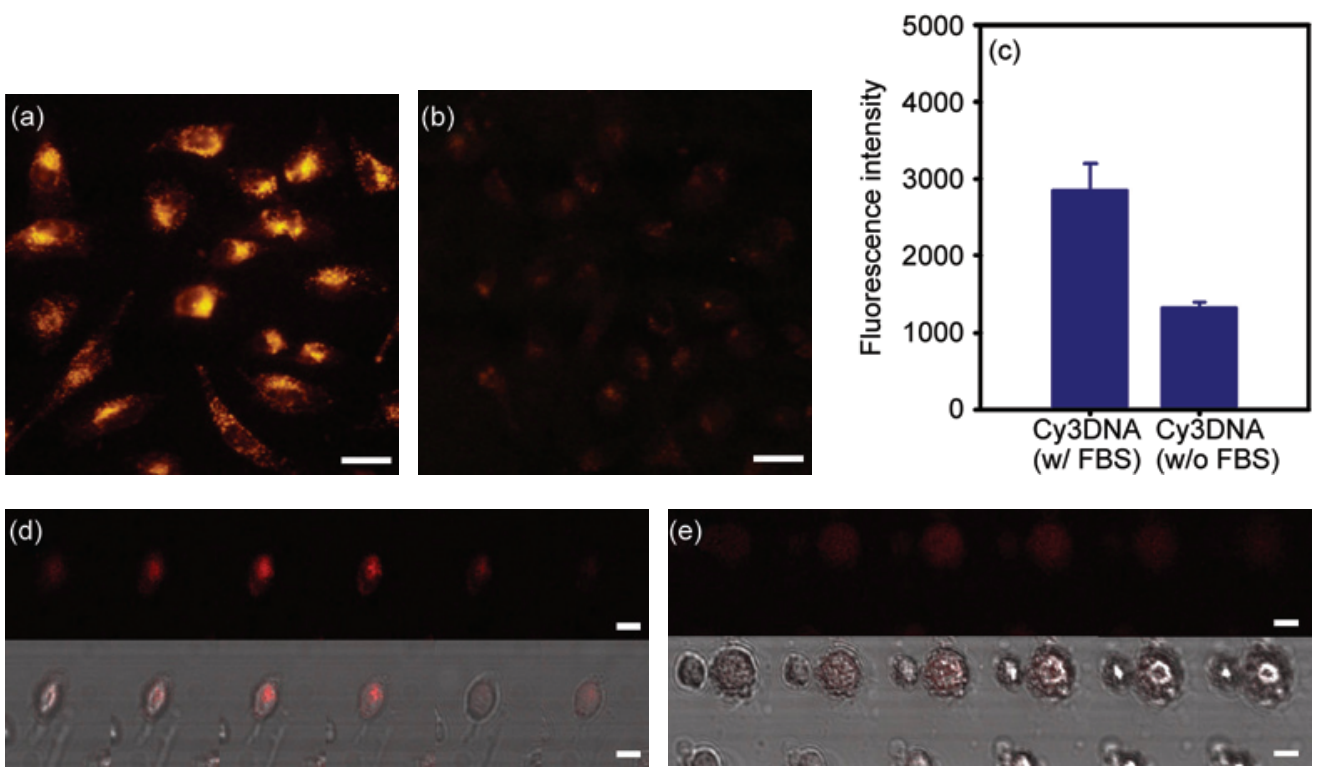

Figure 3 Fluorescence microscopy images of HeLa cells after incubation of Cy3DNA (a) in 10\% FBSsupplemented DMEM, (b) in FBS-free DMEM. The scale bar is $20 \mu \mathrm{m}$. (c) Fluorescence histograms for Cy3DNA incubated in 10\% FBS-supplemented DMEM and in FBS-free DMEM (arithmetic means \pm s.e., $n=3$ ). $2.5 \mu \mathrm{m}$ sectioning confocal fluorescence microscopy images of HeLa cells incubated with Cy3DNA (d) in 10\% FBSsupplemented DMEM and (e) in FBS-free DMEM (upper part: Cy3 emission, 560-610 nm; lower part: overlay of Cy3 fluorescence and transmission image). The scale bar is $10 \mu \mathrm{m}$ 


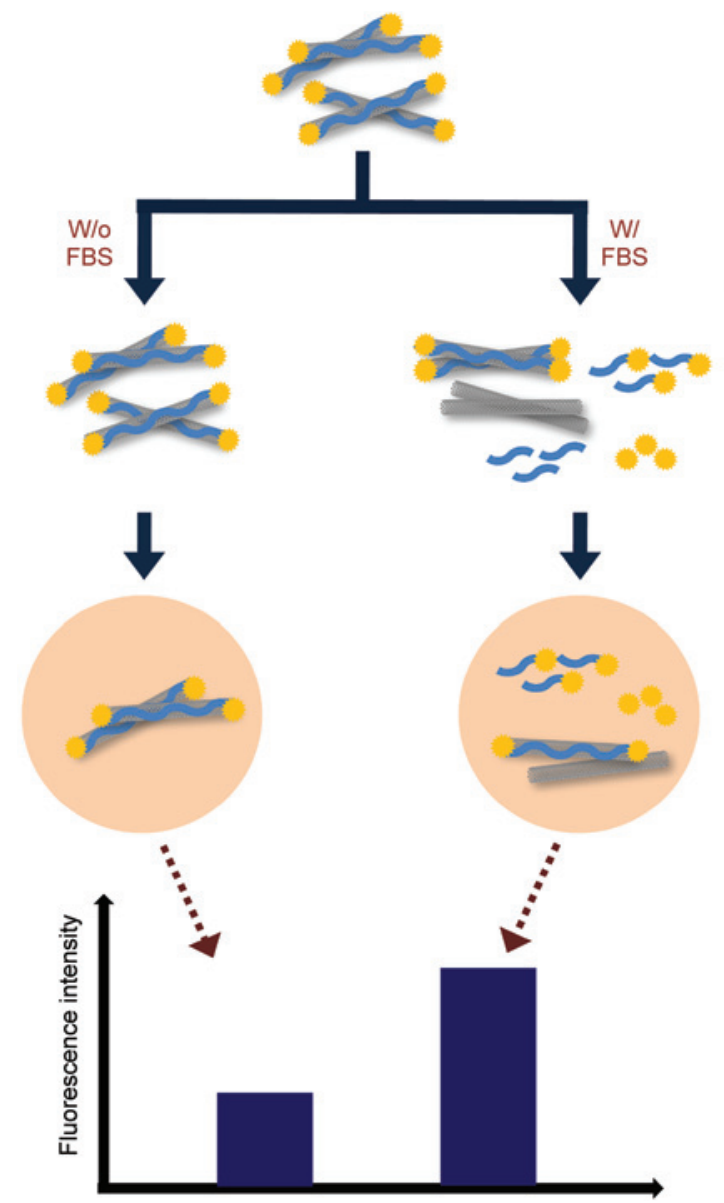

Scheme 1 Schematic representation of the internalization of Cy3DNA-SWNTs in the absence (left path) and presence (right path) of FBS in the incubation medium

the flow cytometry generally provides more accurate cell-based fluorescence intensity as it counts individual cells and simultaneously measures the fluorescence intensity. Figure 5 depicts fluorescenceactivated cell sorter (FACS) histograms obtained from original HeLa cells (Fig. 5(a)), and HeLa cells treated with Cy3DNA-SWNTs (Fig. 5(b)), Cy3DNA (Fig. 5(c)), and $\mathrm{Cy} 3$ dye (Fig. 5(d)) in the presence or absence of nuclease. There is a very marked increase in the mean fluorescence intensity (MFI) for both Cy3DNASWNT (Fig. 5(b)) and Cy3DNA (Fig. 5(c)) treated cells in the presence of FBS. Furthermore, cells treated with Cy3DNA-SWNTs in FBS-free conditions with added S1 nuclease have a very similar high MFI value to that observed in the presence of FBS (Fig. 5(b)). In contrast, a high MFI is observed for Cy3 dye treated cells regardless of the absence or presence of FBS (Fig. 5(d)). These results are in good agreement with the previous cellular image-based fluorescence intensity data.
Additionally, in order to elucidate the actual relative amounts of internalized SWNTs in the presence and absence of FBS, Raman spectroscopy was employed to obtain quantitative information about internalized SWNTs by measuring the $G$ band intensities at $1590 \mathrm{~cm}^{-1}$ [21]. As shown in Fig. 6, the average $G$ band intensities of nine HeLa cells were almost same in the presence and absence of FBS, indicating that the amounts of SWNTs internalized in the cells are similar regardless of the presence of FBS, although the fluorescence intensities are significantly different.
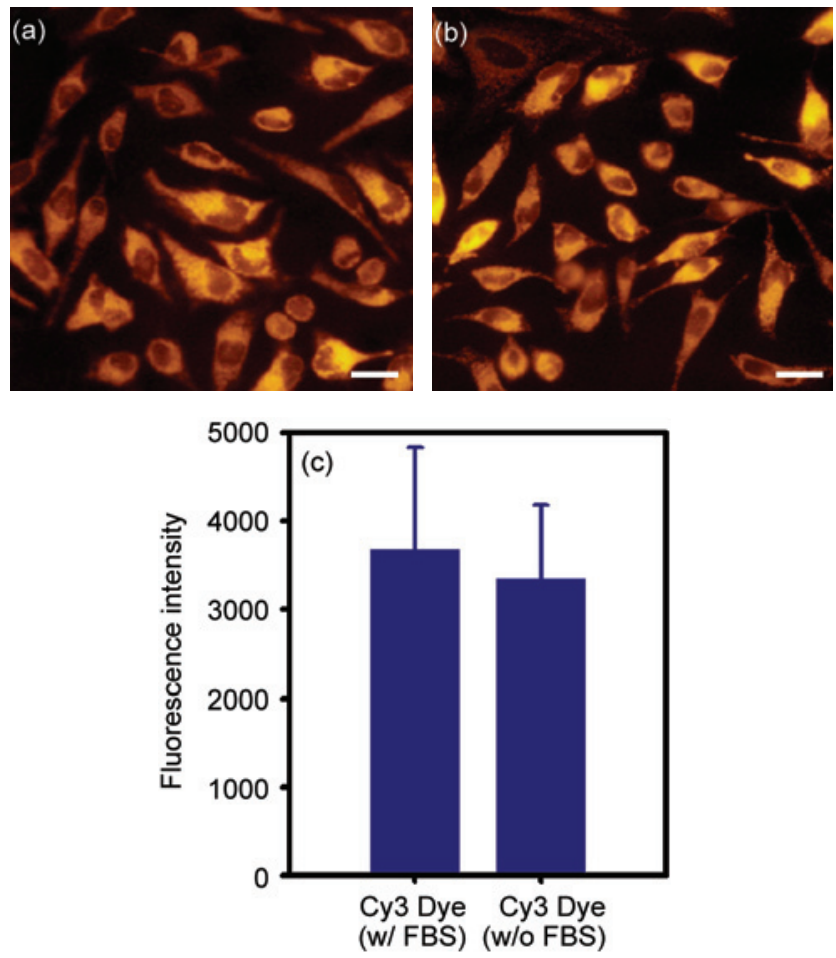

Figure 4 Fluorescence microscopy images of HeLa cells after incubation of Cy3 dye molecules (a) in 10\% FBS-supplemented DMEM and (b) in FBS-free DMEM. The scale bar is $20 \mu \mathrm{m}$. (c) Fluorescence histograms for Cy3 dye molecules incubated in 10\% FBS-supplemented DMEM and in FBS-free DMEM. (arithmetic means \pm s.e., $n=3$ ). The intensities in the presence and absence of FBS are not significantly different (Student's t-test; $P>0.05$ ) 
(a)

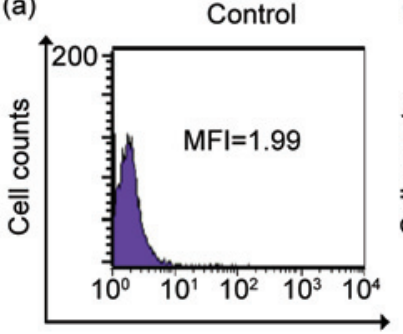

Fluorescence intensity (b)

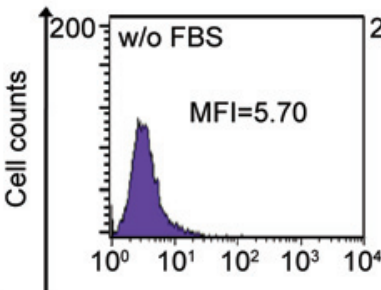

Cy3DNA-SWNT

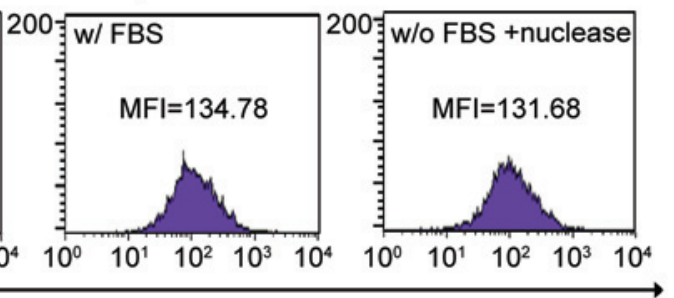

Fluorescence intensity

(c)

Cy3DNA

(d)

Cy3 dye

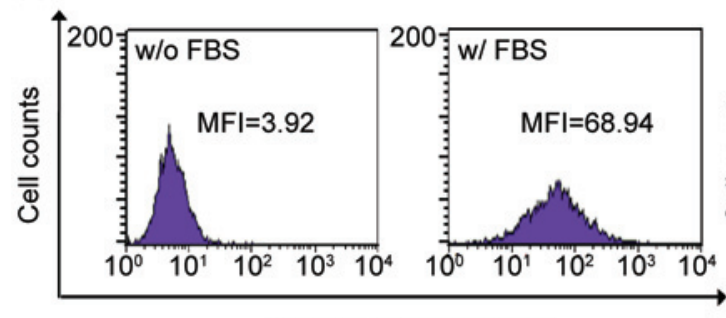

Fluorescence intensity

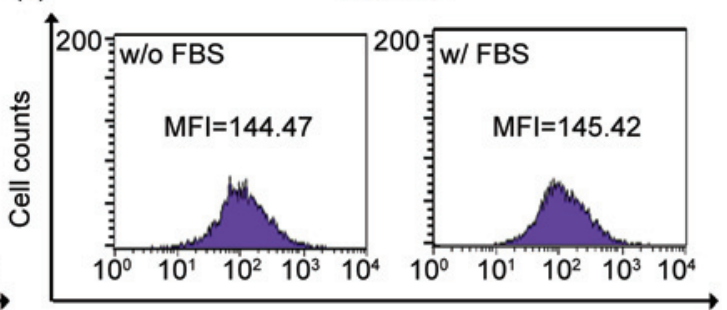

Fluorescence intensity

Figure 5 Flow cytometric histograms showing the cell populations with respect to fluorescence intensities. (a) Untreated HeLa cells, (b) HeLa cells incubated with Cy3DNA-SWNTs in 10\% FBS-supplemented DMEM, in FBS-free DMEM, and FBS-free DMEM with added S1 nuclease. (c) HeLa cells incubated with Cy3DNA in 10\% FBS-supplemented DMEM and in FBS-free DMEM. (d) HeLa cells incubated with Cy3 dye molecules in 10\% FBS-supplemented DMEM and in FBS-free DMEM. The proportion of cells was calculated from the Cy3 fluorescence. MFI indicates the mean fluorescence of total cells
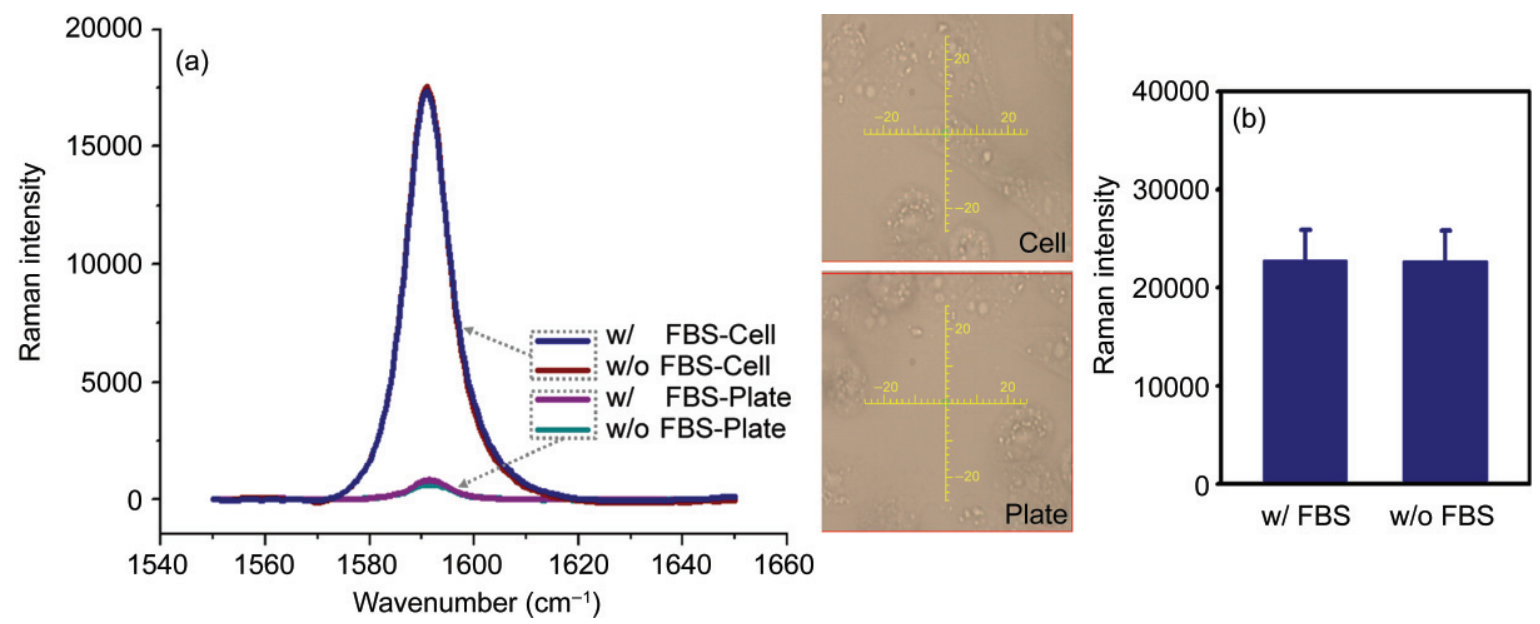

Figure 6 (a) Raman spectra measured from HeLa cells and culture plate after Cy3DNA-SWNT incubation in the presence and absence of FBS (left panel) and optical microscope images of HeLa cells and culture plate (right panel). The laser beam was focused on the green spot (right upper panel: center of a single HeLa cell, right bottom panel: the culture plate). The G band peak intensities at $1590 \mathrm{~cm}^{-1}$ were used to count internalized amounts of SWNTs. (b) Average G band intensities obtained for HeLa cells incubated with Cy3DNA-SWNTs in the presence (left) and absence (right) of FBS in the media (arithmetic means \pm s.e., $n=9$ )

\section{Conclusions}

Cy3 dye fragments are readily released from their original form, Cy3DNA-wrapped SWNTs, in the presence of nucleases during cell internalization. Therefore, it is possible that the fluorescence intensity from cells does not necessarily reflect the actual amount of SWNTs internalized in the cells, and instead is more likely to indicate the amount of free Cy3 dye that is released from Cy3DNA-SWNTs. Hence, an appropriate selection of the cell culture and incubation environment is necessary for systematic 
studies of the inclusion of nanomaterials - including SWNTs - in cells, especially when they are passivated with oligonucleotides.

\section{Acknowledgements}

This work was supported by the Nano/Bio Science \& Technology Program of MOST (2008-00759), Korean Research Foundation (MOEHRD, KRF-2005005-J13103 and KRF-2007-313-C00386), and KOSEF (2007-8-1158).

Electronic Supplementary Material: UV-vis spectra (Fig. S-1), and fluorescence microscopy images and histograms (Figs. S-2 and S-3) can be found in the supplementary material in the online version of this article at http://dx.doi.org/10.1007/s12274-008-8038-z and is accessible free of charge.

\section{References}

[1] Seo, W. S.; Lee, J. H.; Sun, X.; Suzuki, Y.; Mann, D.; Liu, Z.; Terashima, M.; Yang, P. C.; McConnell, M. V.; Nishimura, D. G. et al. FeCo/graphitic-shell nanocrystals as advanced magnetic-resonance-imaging and near-infrared agents. Nat. Mater. 2006, 5, 971-976.

[2] Qian, X.; Peng, X. H.; Ansari, D. O.; Y. G., Q.; Chen, G. Z.; Shin, D. M.; Yang, L.; Young, A. N.; Wang, M. D.; Nie, S. In vivo: Tumor targeting and spectroscopic detection with surface-enhanced Raman nanoparticle tags. Nat. Biotechnol. 2007, 26, 83-90.

[3] Gac, S. L.; Vermes, I.; Berg, A. Quantum dots-based probes conjugated to annexin $\vee$ for photostable apoptosis detection and imaging. Nano Lett. 2006, 6, 1863-1869.

[4] Zheng, G.; Patolsky, F.; Cui, Y.; Wang, W. U.; Lieber, C. M. Multiplexed electrical detection of cancer markers with nanowire sensor arrays. Nat. Biotechnol. 2005, 23, 1294-1301.

[5] Yang, R.; Yang, X.; Zhang, Z.; Zhang, Y.; Wang, S.; Cai, Z.; Jia, Y.; Ma, Y.; Zheng, C.; Lu, Y. et al. Single-walled carbon nanotubes-mediated in vivo and in vitro delivery of siRNA into antigen-presenting cells. Gene Ther. 2006, 13, 1714-1723.

[6] Chen, B. Z.; Wiley, B.; Li, Z. Y.; Campbell, D.; Saeki, F.; Cang, H.; Au, L.; Lee, J.; Li, X.; Xia, Y. Gold nanocages:
Engineering their structure for biomedical applications. Adv. Mater. 2005, 17, 2255-2261.

[7] Klumpp, C.; Kostarelos, K.; Prato, M.; Bianco, A. Functionalized carbon nanotubes as emerging nanovectors for the delivery of therapeutics. Biochem. Biophys. Acta 2006, 1758, 404-412.

[8] Panchapakesan, B.; Lu, S.; Sivakumar, K.; Teker, K.; Cesarone, G.; Wickstrom, E. Single wall carbon nanotube nanobomb agents for killing breast cancer cells. NanoBiotechnology 2005, 1, 133-140.

[9] Shao, N.; Lu, S.; Wickstrom, E.; Panchapakesan, B. Integrated molecular targeting of IGF1R and HER2 surface receptors and destruction of breast cancer cells using single wall carbon nanotubes. Nanotechnology 2007, 18, 315101.

[10] Kam, N. W.; O'Connell, M.; Wisdom, J. A.; Dai, H. Carbon nanotubes as multifunctional biological transporters and near-infrared agents for selective cancer cell destruction. Proc. Natl. Acad. Sci. U.S.A. 2005, 102, 11600-11605.

[11] Kam, N. W.; Jessop, T. C.; Wender, P. A.; Dai, H. Nanotube molecular transporters: Internalization of carbon nanotube-protein conjugates into mammalian cells. J. Am. Chem. Soc. 2004, 126, 6850-6851.

[12] Pantarotto, D.; Singh, R.; McCarthy, D.; Erhardt, M.; Briand, J. P.; Prato, Maurizio.; Kostarelos, K.; Bianco, A. Functionalized carbon nanotubes for plasmid DNA gene delivery. Angew. Chem. Int. Ed. 2004, 43, 5242-5246.

[13]Cai, D.; Mataraza, J. M.; Qin, Z.-H.; Huang, Z.; Huang, J.; Chiles, T. C.; Carnahan, D.; Kempa, K.; Ren, Z. F. Highly efficient molecular delivery into mammalian cells using carbon nanotube spearing. Nat. Methods 2005, 2, 449454.

[14]Bianco, A.; Hoebeke, J.; Godefroy, S.; Chaloin, O.; Pantarotto, D.; Briand, J. P.; Muller, S.; Prato, M.; Partidos, C. D. Cationic carbon nanotubes bind to CpG oligodeoxynucleotides and enhance their immunostimulatory properties. J. Am. Chem. Soc. 2005, 127, 58-59.

[15] Liu, Ye.; Wu, D. C.; Zhang, W.-D.; Jiang, X.; He, C.-B.; Chung, T. S.; Goh, S. H.; Leong, K. W. Polyethyleniminegrafted multiwalled carbon nanotubes for secure noncovalent immobilization and efficient delivery of DNA. Angew. Chem. Int. Ed. 2005, 44, 4782-4785.

[16]Lu, Q.; Moore, J. M.; Huang, G.; Mount, A. S.; Rao, A. M.; Larcom, L. L.; Ke, P. C. RNA polymer translocation with single-walled carbon nanotubes. Nano Lett. 2004, 4 , 2473-2477. 
[17]Kam, N. W.; Liu, Z.; Dai, H. Functionalization of carbon nanotubes via cleavable disulfide bonds for efficient intracellular delivery of siRNA and potent gene silencing. J. Am. Chem. Soc. 2005, 127, 12492-12493.

[18]Zhang, Z.; Yang, X.; Zhang, Y.; Zeng, B.; Wang, S.; Zhu, T.; Roden, R. B. S.; Chen, Y.; Yang, R. Delivery of telomerase reverse transcriptase small interfering RNA in complex with positively charged single-walled carbon nanotubes suppresses tumor growth. Clin. Cancer Res. 2006, 12, 4933-4939.

[19]Becker, M. L.; Fagan, J. A.; Gallant, N. D.; Bauer, B. J.; Bajpai, V.; Hobbie, E. K.; Lacerda, S. H.; Migler, K. B.; Jakupciak, J. P. Length-dependent uptake of DNAwrapped single-walled carbon nanotubes. Adv. Mater 2006, 19, 939-945.

[20] Piva, R.; Lambertini, E.; Penolazzi, L.; Facciolo, M. C.; Lodi, A.; Aguiari, G.; Nastruzzi, C.; Senno, L. D. In vitro stability of polymerase chain reation-generated DNA fragments in serum and cell extracts. Biochem. Pharmacol. 1998, 56, 703-708.

[21] Kawamoto, H.; Uchida, T.; Kojima, K.; Tachibana, M. $\mathrm{G}$ band Raman features of DNA-wrapped single-wall carbon nanotubes in aqueous solution and air. Chem. Phys. Lett. 2006, 432, 172-176.
[22 ] Sánchez-Pomales, G.; Morales-Negrón, Y.; Cabrera, C. R. Study of self-assembled monolayers of DNA and DNAcarbon nanotube hybrids. Rev. Adv. Mater. Sci. 2005, 10, 261-265.

[23]Uchida,T.; Kumar, S. Single wall carbon nanotube dispersion and exfoliation in polymers. J. Appl. Polym. Sci. 2005, 98, 985-989.

[24]O'Connell, M. J.; Bachilo, S. M.; Huffman, C. B.; Moore, V. C.; Strano, M. S.; Haroz, E. H.; Rialon, K. L.; Boul, P. J.; Noon, W. H.; Kittrell, C. et al. Band gap fluorescence from individual single-walled carbon nanotubes. Science 2002, 297, 593-596.

[25] Zheng, M.; Jagota, A.; Semke, E. D.; Diner, B. A.; Mclean, R. S.; Lustig, S. R.; Richardson, R. E.; Tassi, N. G. DNAassisted dispersion and separation of carbon nanotubes. Nat. Mater. 2003, 2, 338-342.

[26]Patil, S.; Sandberg, A.; Heckert, E.; Self, W.; Seal, S. Protein adsorption and cellular uptake of cerium oxide nanoparticles as a function of zeta potential. Biomaterials 2007, 28, 4600-4607.

[27]Chithrani, B. D.; Ghazani, A. A.; Chan, W. C. W. Determining the size and shape dependence of gold nanoparticle uptake into mammalian cells. Nano Lett. 2006, 6, 662-668. 